\title{
Efeitos de doses elevadas da terbinafina e itraconazol em ratos Wistar
}

\author{
Ana Raquel Mano Meinerz*,1, Marlete Brum Cleff', Patrícia da Silva Nascente ${ }^{2}$, \\ Márcia de Oliveira Nobre ${ }^{3}$, Luiz Filipe Damé Schuch², Tatiana de Ávila Antunes², \\ Melissa O. Xavier², Mário Carlos Araújo Meireles², João Roberto de Braga Mello ${ }^{1}$
}

\begin{abstract}
${ }^{1}$ Departamento de Veterinária Preventiva, Faculdade de Veterinária, Universidade Federal do Rio Grande do Sul, ${ }^{2}$ Departamento de Veterinária Preventiva, Universidade Federal de Pelotas, ${ }^{3}$ Departamento de Clínica Veterinária, Universidade Federal de Pelotas
\end{abstract}

*Correspondência:

A. R. M. Meinerz

Departamento de Veterinária

Preventiva

Universidade Federal de Pelotas Caixa Postal 354 - Campus

Universitário

96010-900 - Pelotas - RS, Brasil

E-mail: rmeinerz@bol.com.br
Levando em consideração a importância do itraconazol e da terbinafina na terapia antifúngica, o estudo tem como objetivo avaliar os efeitos de altas doses de itraconazol (100 mg/kg) e terbinafina $(250 \mathrm{mg} / \mathrm{kg})$ em ratos albinos wistar através das análises das enzimas hepáticas (ALT e ALP), hemograma completo e estudo histopatológico de diferentes órgãos. Os fármacos foram administrados pela via oral, uma vez ao dia, por um periodo de 30 dias, quando foi coletado sangue e realizado a necrópsia dos animais experimentais. Os valores detectados das enzimas hepáticas e do hemograma foram compativeis aos indices fisiológicos para a espécie estudada não sendo observadas diferenças estatísticas entre os grupos experimentais. A histopatologia não revelou nenhuma anormalidade, porém $25 \%$ dos animas tratados com terbinafina morreram imediatamente após a administração do fármaco. Com esses resultados concluiu-se que as doses administradas dos fármacos não alteraram as enzimas hepáticas avaliadas, assim como o hemograma, sendo, no entanto, necessários mais estudos que possibilitem considerar doses elevadas da terbinafina e itraconazol como alternativa terapêutica viável para o tratamento de micoses sistêmicas.

\section{INTRODUÇÃO}

Os fármacos antifúngicos disponíveis são, ainda, em número limitado, sendo que as principais famílias de antifúngicos compreendem os poliênicos, azólicos, tiocarbamatos, alilaminas, derivados morfolínicos, 5fluorcitosina e griseofulvina. Os agentes antifúngicos, na sua maioria, produzem efeitos tóxicos, devido aos fungos compartilharem, com a célula do hospedeiro humano, o caráter eucariota, com conseqüentes similaridades bioquímicas e fisiológicas, que limitam muito o arsenal terapêutico (Odds, 2003; Catalán, Montejo, 2006). Os iodetos foram as primeiras substâncias utilizadas na terapia antifúngica, tanto nos homens quanto nos animais, especialmente no tratamento da esporotricose, representando uma alternativa eficaz e de baixo custo. Porém, os preparados contendo iodo tiveram seu uso limitado devido aos freqüentes efeitos tóxicos, especialmente na espécie felina (Coskun et al., 2004). 
O itraconazol, antifúngico do grupo dos triazóis, emergiu como uma alternativa terapêutica eficaz, com reduzidos efeitos colaterais em relação aos outros antifúngicos, sendo introduzido, em 1992, para o tratamento da aspergilose, histoplasmose e blastomicose, ampliando, posteriormente, o seu espectro de ação frente a diversas espécies de fungos patogênicos. As vantagens relacionadas ao fármaco levaram ao intenso uso, sendo, atualmente, um dos antifúngicos mais utilizados nas clínicas médicas humana e veterinária. Entretanto, devido, principalmente, ao seu uso indiscriminado têm sido observados freqüentes relatos de isolados fúngicos resistentes, conseqüentemente falhas terapêuticas e remissão de enfermidades micóticas (Kauffman et al., 2000; Pierad et al., 2000; Rochette et al., 2003; Schubach et al., 2004; Catalán, Montejo, 2006).

A terbinafina, antifúngico do grupo das alilaminas, tem amplo espectro de ação in vitro e in vivo frente a fungos responsáveis por micoses superficiais e sistêmicas. O fármaco apresenta natureza lipofílica e queratofílica, o que leva ao seu acúmulo no tecido adiposo e queratinoso. Devido a essas características é considerado o antifúngico de eleição para o tratamento das dermatofitoses e onicomicoses (Hay, 1999; Perez, 1999; Ryder, 1999; Jessup et al., 2000; Darkes et al., 2003).

O itraconazol e a terbinafina possuem entre si particularidades relacionadas ao mecanismo de ação e toxicidade que devem ser consideradas. Ambos agem inibindo enzimas envolvidas com a síntese do ergosterol, porém a terbinafina atua mais precocemente na cadeia da biossíntese tendo um efeito primário fungicida, enquanto o itraconazol provoca a inibição de enzimas dependentes do citocromo P-450, com efeito, primariamente fungistático. A interação do itraconazol com o citocromo $\mathrm{P}-450$ pode causar alterações nas enzimas hepáticas e a inibição do sistema enzimático dos mamíferos, como os envolvidos com a síntese dos hormônios esteróides e prostaglandinas, que são dependentes deste citocromo (Doncker, 1999; Jaham et al., 2000; Pierad et al., 2000).

Os principais efeitos colaterais descritos para terbinafina e itraconazol envolvem, principalmente, o sistema gastrintestinal, sendo os mais comumente relatados náuseas, vômitos, dor e desconforto abdominal. Devido aos fármacos serem metabolizados no fígado, podem ser observadas alterações nas enzimas hepáticas. Estudos demonstraram que a concentração hepática do itraconazol é até três vezes maior que os níveis plasmáticos, sendo recomendada a monitoração das enzimas hepáticas durante o tratamento com o fármaco (Amichai, Grunwald, 1998; Catalán, Montejo, 2006).

Considerando a importância do itraconazol e terbinafina na terapia antifúngica, assim como o reduzido número de antifúngicos disponíveis para o tratamento das micoses sistêmicas, o estudo teve como objetivo avaliar os efeitos desses fármacos em animais experimentais administrando doses maiores do que as recomendadas.

\section{MATERIAIS E MÉTODOS}

Foram utilizados 40 ratos norvergicus (Rattus rattus), albinos, linhagem Wistar, com oito semanas de idade, machos e com peso médio de $300 \mathrm{~g}$. Os animais foram alojados no Biotério Central da Universidade Federal de Pelotas (UFPel), sendo mantidos em condições constantes de umidade, temperatura e ciclo de claro e escuro, recebendo dieta de acordo com o peso corporal e água "ad libitum" durante o período experimental.

Os animais foram distribuídos em quatro grupos experimentais: Grupo tratado terbinafina (os quais foram administrados $250 \mathrm{mg} / \mathrm{kg}$ de terbinafina diluída em uma solução contendo $1 \%$ de Tween 20 e 5\% de DMSO); grupo tratado itraconazol (administrando $100 \mathrm{mg} / \mathrm{kg}$ de itraconazol diluído em água destilada estéril) e os grupos diluente terbinafina e diluente itraconazol, em que foram administrados os respectivos diluentes de cada fármaco.

A administração dos fármacos e diluentes foi realizada uma vez ao dia, pela via oral com o auxilio de uma sonda orogástrica, durante o período de 30 dias, em todos os animais experimentais. Durante o estudo, os animais foram avaliados, a fim de observar alterações físicas e/ou comportamentais.

No final do período experimental foi coletado sangue através de punção-cardíaca, para a realização do hemograma completo e perfil hepático. As enzimas hepáticas (Alanina aminotransferase-ALT e Fosfatase alcalinaALP) foram analisadas através do Kit comercial Lab-test, executado de acordo com as normas do fabricante, com a posterior leitura em aparelho espectrométrico. Os exames laboratoriais foram realizados no laboratório de Patologia Clínica da Faculdade de Veterinária da Universidade Federal de Pelotas-UFPel.

Após a coleta de sangue, os animais foram sacrificados através de anestesia profunda e necropsiados, sendo coletados órgãos do sistema digestório, rins, gânglios e tecido subcutâneo. Os órgãos colhidos para o exame histopatológico foram acondicionados em formol a $10 \%$, sendo, posteriormente, incluídos em parafina e processados, utilizando-se coloração de HE (Hematoxilina-Eosina) e PAS (Ácido Periódico-Schiff), para posterior análise histopatológica.

Foi realizada análise de variância através do teste ANOVA e a comparação entre médias pelo teste de Tukey a 0,05 de significância, utilizando o programa Statistix 8.0, 
para estimar as alterações referentes ao hemograma completo, assim como das enzimas hepáticas avaliadas, nos animais submetidos ao tratamento antifúngico.

\section{RESULTADOS E DISCUSSÃO}

Os resultados referentes às análises da Alanina Aminotransferase (ALT) e Fosfatase Alcalina (ALP) nos animais pertencentes a todos os grupos experimentais, apresentaram valores dentro dos limites normais para a espécie estudada (Harkness, Wagner, 1995; Farias et al., 2000), não existindo diferenças significativas entre os tratamentos, confirmado pelo teste de Tukey a 0,05 de significância (Tabela I). Em relação à análise histopatológica dos órgãos e tecidos coletados para necrópsia, não foram evidenciadas quaisquer alterações, em todos os grupos experimentais avaliados, assim como nos gânglios analisados (Figura 1).

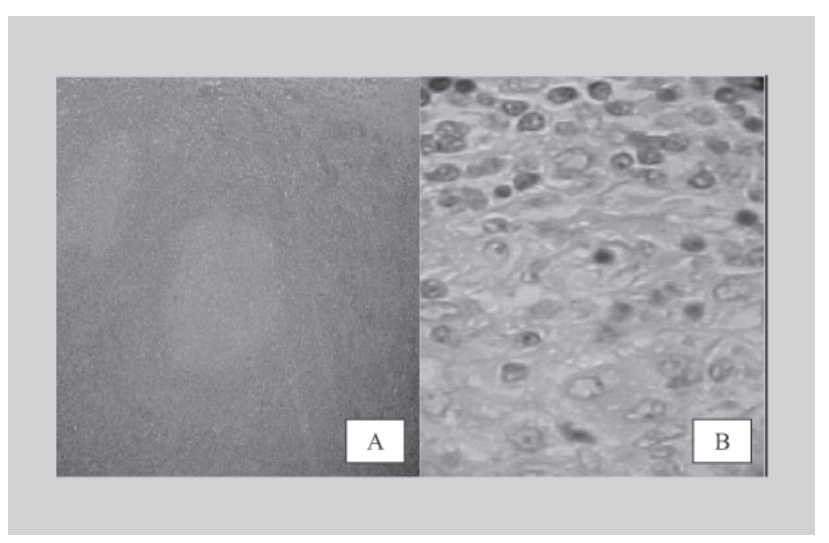

FIGURA 1 - Corte histológico de linfonodo poplíteo de animais do grupo tratado itraconazol com presença de linfócitos, macrófagos e fibroblastos em coloração de PAS (100X-A e 400X-B).

As doses preconizadas para terbinafina no tratamento de micoses superficiais correspondem a $250 \mathrm{mg}$, enquanto que para o itraconazol são de $100 \mathrm{mg}$ a $400 \mathrm{mg} /$ dia para micoses cutâneas e sub-cutâneas (De Pauw et al., 2000). Neste estudo, as altas doses administradas dos fármacos não provocaram alteração na função hepática, assim como não produziram efeitos tóxicos para os hepatócitos durante o período estudado.

Em se tratando da terbinafina, tem-se descrito relação entre as concentrações disponíveis do fármaco conforme o diluente utilizado. Kan e Bennet (1988) observaram que a terbinafina diluída em uma solução com $1 \%$ Tween 20 e 5\% DMSO em água destilada resultou em maiores níveis plasmáticos do fármaco. Estudos têm demonstrado que a ação da terbinafina é dose-dependente, alcançando concentrações maiores no plasma quando administrada em doses elevadas (Hosseini-Yeganeh, McLachlan, 2002; Chapman et al., 2004). O efeito semelhante tem sido observado com o itraconazol, que, quando administrado em doses maiores, resulta no aumento das concentrações plasmáticas, ocorrendo, conseqüentemente, uma ação mais efetiva do fármaco. O declínio dos níveis plasmáticos a valores inferiores a $6 \mu \mathrm{g} / \mathrm{mL}$ foi relacionado com a redução da atividade e a falhas terapêuticas (Pierard et al., 2000).

Os efeitos hepatotóxicos provocados pela terbinafina são de rara ocorrência, sendo que a elevação da ALT, AST e da bilirrubina ocorre em menos de $10 \%$ dos pacientes. Embora disfunção hepática não seja um sinal clinico relevante de intoxicação, têm sido relatados casos de pacientes que desenvolveram hepatotoxicidade, com o aumento transitório das enzimas hepáticas, normalizando com a interrupção do tratamento (Amichai, Grunwald, 1998; Brustein et al., 2004).

Em relação ao itraconazol, o aumento temporário das enzimas hepáticas é o efeito colateral mais freqüentemente descrito em humanos, sendo geralmente, reversível e transitório. Porém, o uso de altas doses por um período prolongado aumenta a eliminação do fármaco no soro, o que pode contribuir para a toxicidade hepática. (Amichai, Grunwald, 1998; Pierard et al., 2000; Odds, 2003).

Quanto aos resultados correspondentes ao hemograma, eritrograma (hematócrito, hemoglobina e contagem de hemácia) e leucograma (leucócitos, neutrófilos e linócitos) resultaram em níveis fisiológicos para a espécie estudada (Harkness, Wagner, 1995), tanto em relação à terbinafina como para o itraconazol (Tabela II), não havendo diferenças significativas entre os tratamentos, confirmado pelo teste de Tukey a 0,05 de significância.

TABELA I - Valores referentes aos níveis da Alanina aminotransferase (ALT) e Fosfatase Alcalina (FA) dos animais experimentais submetidos a administração dos fármacos antifúngicos e seus respectivos diluentes

\begin{tabular}{lcccc}
\hline Enzimas avaliadas & TT & DT & TI & DI \\
\hline ALT (UI/L) & $30-36$ & $20-42$ & $26-45$ & $29-45$ \\
ALP (UI/L) & $39,2-50,7$ & $38-72,1$ & $40-50,7$ & $42,4-64,3$ \\
\hline
\end{tabular}

TT-tratado terbinafina; DT-diluente terbinafina; TI-tratado itraconazol; DI-diluente itraconazol. 
TABELA II - Valores referentes a série branca e série vermelha dos animais experimentais submetidos à administração dos fármacos antifúngicos e seus respectivos diluentes

\begin{tabular}{lcccc}
\hline Parâmetros avaliados & TT & DT & TI & DI \\
\hline Contagem de células vermelhas $(\mathrm{x} 10 \mu \mathrm{L})$ & $6,6-7,0$ & $7,0-7,5$ & $6,5-8,0$ & $6,5-7,5$ \\
Hemoglobina $(\mathrm{g} / \mathrm{dL})$ & $12-15$ & $12,5-14$ & $14-15$ & $13,5-15$ \\
Hematócrito $(\%)$ & $38-40$ & $39-40$ & $38-42$ & $38-40$ \\
Plaquetas $(\mathrm{x} 10 \mu \mathrm{L})$ & $650-720$ & $660-700$ & $600-730$ & $640-700$ \\
Contagem de células brancas $(\mathrm{x} 10 \mu \mathrm{L})$ & $6,0-9,0$ & $7,0-9,0$ & $6,0-9,5$ & $6,5-9,0$ \\
Neutrófilos $(\mathrm{x} 10 \mu \mathrm{L})$ & $2,0-3,0$ & $2,5-3,0$ & $1,9-3,2$ & $2,0-3,5$ \\
Linfócitos $(\mathrm{x} 10 \mu \mathrm{L})$ & $6,0-7,5$ & $6,5-7,0$ & $6,5-8,0$ & $6,0-7,0$ \\
Volume de sangue total $(\mathrm{mL} / \mathrm{kg})$ & $55-60$ & $58-60$ & $56-59$ & $55-63$ \\
\hline
\end{tabular}

TT-tratado terbinafina; DT-diluente terbinafina; TI-tratado itraconazol; DI-diluente itraconazol

Durante o período experimental foram evidenciadas alterações físicas no grupo tratado-terbinafina como: distensão abdominal, salivação prévia à administração do fármaco, redução de apetite e agressividade. No decorrer do estudo, $25 \%(5 / 20)$ destes animais vieram ao óbito imediatamente após sondagem oral, não sendo evidenciadas alterações na análise histopatológica. Provavelmente, os óbitos ocorreram devido aos efeitos colaterais descritos para o fármaco, o que poderia favorecer a falsa-via na passagem da sonda. A mudança de comportamento, provavelmente, foi devida ao desconforto provocado pela ação do fármaco. Kan e Bennet (1988) também registraram a morte por falsa-via em animais experimentais quando administraram terbinafina associada aos mesmos diluentes utilizados neste estudo.

\section{CONCLUSÃO}

A administração de altas doses de terbinafina e itraconazol não ocasionou alterações no hemograma, nas enzimas hepáticas e no exame histopatológico dos animais experimentais, necessitando, no entanto, análises complementares que contribuam aos avanços dos estudos da terapia antimicótica, especialmente nas micoses sistêmicas, em que altas doses de fármacos antifúngicos são requeridas.

\section{ABSTRACT \\ Effects of high doses of terbinafine and itraconazole in Wistar rats}

Taking into account the importance of the itraconazole and terbinafine in the antifungal therapy, the study has as objective evaluate the effects of high itraconazole doses $(100 \mathrm{mg} / \mathrm{kg})$ and terbinafine $(250 \mathrm{mg} / \mathrm{kg})$ in rats albino Wistar through the analyses of the hepatic enzymes (ALT and ALP), complete blood count and histopathologic study of different organs. The drugs were administered orally once a day, for a period of 30 days, when blood was collected and accomplished the necropsy of the experimental animals. The detected values of the hepatic enzymes and of the blood count were compatible to the physiologic indexes for the studied species with no statistical differences among the experimental groups. The histopathologic exam did not reveal any abnormality, however $25 \%$ of the treated with terbinafine died immediately after the administration of the drug. With those results we concluded that the administered doses of the drugs did not alter the appraised hepatic enzymes, as well as the blood count. However, more studies are needed to consider high doses of the terbinafine and itraconazole as viable treatment alternative for systemic mycosis.

UNITERMS: Antifungal. Itraconazole. Terbinafine. Mycosis.

\section{REFERÊNCIAS}

AMICHAI, B; GRUNWALD, M. H. Adverse drug reactions of the new oral antifungal agents-terbinafine, fluconazole and itraconazole. Int. J. Dermat., v. 37, p. 410-415,1998.

BURSTEIN, Z; VILDÓSOLA, H; LOZANO, Z; VERONA, R; VARGAS, G. Hepatitis tóxica colestásica por terbinafina: reporte de caso. Rev. Gastroenter., v. 24, p. 357-362, 2004 .

CATALÁN, M.; MONTEJO, J. C. Antifúngicos sistémicos. Rev. Iberoam. Micol., v. 23, p. 39-49, 2006.

DE PAUW, B. E. New antifungal agents and preparation. Inter. J. Antimicrob. Agents, v. 16, p. 147-150, 2000. 
CHAPMAN, S. W; PAPPAS, P; KAUFFMAN, C; SMITH, E. B; DIETZE, R; TIRABOSCHI-FOSS, N; RESTREPO, A; BUSTAMANTE, A B; OPPER, C; EMADY-AZAR, S; BACHI, R. Comparative evaluation of the efficacy and safety of two doses of terbinafine (500 and $1000 \mathrm{mg}$ /day) in the treatment of cutaneous or lymphocutaneous sporotrichosis. Mycoses, v. 47, p. $62-$ 68, 2004.

COSKUN, B; SARAL, Y; AKPOLAT, N; ATASEVEN, A; ÇICEK, D. Sporotrichosis successfully treated with terbinafine and potassio iodide: case report and review of the literature. Mycopathologia, v. 158, p. 53-56, 2004.

DARKES M. J, SCOTT, L. J, GOA, K. L. Terbinafine: a review of its use in onychomycosis in adults. Am. J. Clin. Dermatol., v. 4, n. 1, p. 39-65, 2003.

DONCKER, P. Itraconazole and terbinafine in pespective: from petri dish. J. Eur. Acad. Dermat. Veneorol., v. 12, p. 10-16, 1999.

FARIAS, E. A.; FONTES, P. R. O.; RHODEN, C. R.; LUCAS, M. L.; LEAL, M. L. M.; SABEDOTTI, E. L. A. Acompanhamento de um modelo de indução de cirrose em ratos mediante a vídeolaparoscopia. Acta Cirurg. Bras., v. 15, p. 163-167, 2000.

HARKNESS, J. E.; WAGNER, J. E. The biology and medicine of rabbits and rodents. Baltimore:Williams and Williams, $1995.372 \mathrm{p}$.

HAY, R. J. Therapeutic potential of terbinafine in subcutaneous and systemic mycoses. Br. J. Dermatol., v. 141, p. 36-40, 1999.

HOSSEINI-YEGANEH, M.; McLACHLAN, A. Physiologically based pharmacokinetics model for terbinafine in rats and humans. Antimicrob. Agents Chemother, v. 46, p. 2219-2228, 2002.

JAHAM, C.; PARADES, M.; PAPICH, M. G. Antifungical dermatologic agents: azoles and alllylamines. Anim. exot. small mag., v.22, n.6, p.548-558, 2000.
JESSUP, C. J.; RYDER, N. S.; GHANNOUM, M. A. An evaluation of the in vitro of terbinafine. Med. Micol., v.38, n.2, p. 155-159, 2000.

KAN, V. L.; BENNETT, J. E. Efficacies of four antifungal agents in experimental murine sporotrichosis. Antimicrob. Agents Chemother., v. 32, n. 11, p. 1619-23, 1988.

KAUFFMAN, C. A..; HAJJEH, R.; CHAPMAN, S. W. Pratice Guidelines for the management of patients with sporotrichosis. Clin. Infec. Dis., v. 30, p. 684-687, 2000.

ODDS, F. C. Antifungal agents: their diversity and increasing sophistication. Mycologist, v. 17, p. 51-55, 2003.

PEREZ, A. Terbinafine: broad new spectrum of indications in several subcutaneous and systemic and parasitic diseases. Mycoses, v. 42, p. 111-114, 1999.

PIERARD, G. E.; ARRESE, J. E.; PIERARDFRANCHIMONT, C. Itraconazole. Expert Opin. Pharmacother., v. 1, n. 2, p. 287-304, 2000.

ROCHETTE, F; ENGELEN, M; VANDEN BOSSCHEM, $\mathrm{H}$. Antifungal agents of use in animal health-pratical applications. J. Vet. Pharmacol., v. 26, p. 31-53, 2003.

RYDER, N. S. Activity of terbinafine against serious fungal pathogens. Mycoses, v. 42, p. 115-119, 1999.

SCHUBACH T. M. P, SCHUBACH, A. O, KAMOTO BARROS, T. M. B. L, FIGUEIREDO, F. B, CUZZI, T, FIALHO-MONTEIRO, P. C, REIS, R. S; PEREZ, M. A. Evaluation of an epidemic of sporotrichosis in cats: 347 cases (1998-2001). J. Am. Vet. Med. Assoc., v. 224, p. 1623-1629, 2004.

Recebido para publicação em 30 de julho de 2006. Aceito para publicação em 29 de março de 2007. 